\title{
Non-psychotic, non-major affective psychiatric disorders in adolescent boys were associated with greater schizophrenia risk
}

\author{
Weiser M, Reichenberg A, Rabinowitz J, et al. Association between nonpsychotic psychiatric diagnoses in adolescent males \\ and subsequent onset of schizophrenia. Arch Gen Psychiatry 2001 Oct;58:959-64.
QUESTION: In adolescent boys with a non-psychotic, non-major affective psychiatric disorder, what is the risk of future schizophrenia?

Design

Population based cohort study with follow up of 4-8 years.

\section{Setting}

Israel.

\section{Patients}

9365 boys who had a non-psychotic, non-major affective psychiatric disorder from a cohort of 124244 boys aged 16-17 years who had a mandatory medical and psychiatric health assessment by the Israeli draft board. Adolescents with affective disorders and those who were admitted to hospital for schizophrenia prior to or within 1 year after the draft board assessment were excluded from the analysis.
Source of funding: Stanley Foundation.

For correspondence: Dr M Davidson, Chaim Sheba Medical Center, Tel-Aviv University, Tel-Hashomer, Israel. davidso@netvision.net.il

\section{Assessment of prognostic factors}

The mental health assessment included diagnoses of schizophrenia spectrum personality disorders, adjustment disorder, antisocial personality disorder, impulse control disorder, mental retardation, alcohol and drug abuse, neurosis, and other personality disorders.

\section{Main outcome measures}

The National Psychiatric Hospitalisation Case Registry was used to identify those adolescents screened by the draft board who were later admitted to hospital for schizophrenia.

\section{Main results}

96 adolescent boys (1.03\%) were admitted to hospital 4-8 years later for schizophrenia. The prevalence of non-psychotic non-major affective psychiatric disorders was higher in patients who developed schizophrenia than in the general population of adolescents $(26.8 \% \mathrm{v}$ $7.4 \%$, odds ratio $4.5,95 \%$ CI 3.6 to 5.6 ). The various non-psychotic psychiatric disorders were associated with different risks of hospital admissions for schizophrenia. The table presents odds ratios for having various premorbid disorders in adolescents with schizophrenia compared with the general population of adolescents.

\section{Conclusion}

In adolescent boys, a non-psychotic, non-major affective psychiatric disorder was associated with an increased risk of schizophrenia.

\section{COMMENTARY}

In many people, psychiatric problems often precede schizophrenia. This prodromal phase may lead to psychiatric consultation and can pose diagnostic difficulties. In the study by Weiser et al, an association between non-psychotic psychopathology and the later development of schizophrenia was found.

The opportunity to link data from conscription with psychiatric admission provided an ideal design for answering this research question. Psychopathology was assessed during a compulsory health screening for 16-17 year old boys. The screening diagnosis was done before the onset of schizophrenia and was therefore unbiased in relation to the disease, although concerns will remain over the reliability of diagnosis at screening, particularly for personality disorder. The follow up identified all schizophrenia admissions in Israel. The authors were unable to include people with schizophrenia who were not admitted to hospital, which is probably not a serious omission. The analysis only adjusted for intelligence quotient score, and it would have been preferable if other potential confounders had also been adjusted for.

The study therefore confirms the findings of the Swedish survey ${ }^{1}$ that also reported an increase in non-psychotic disorders before the onset of schizophrenia. In the Swedish study, $38 \%$ of participants who subsequently developed schizophrenia had a psychiatric diagnosis at 18 years of age. In this study by Weiser et al, $27 \%$ of participants had a psychiatric diagnosis. The younger age range (16-17 y) might explain this difference, but differences in the pattern of diagnoses were also present. In the Swedish ${ }^{1}$ and Weiser et al studies, the prevalence of neurosis was $5 \%$ and $1.1 \%$, respectively, and the prevalence of personality disorder was $2.6 \%$ and $5.6 \%$, respectively.

How does one interpret these findings? The observation that non-psychotic psychiatric problems are common before schizophrenia appears robust and accords with other evidence. The observation that paranoid and schizotypal personality disorders increase the risk of schizophrenia also appears to be consistent with past research. Of more interest is the possibility that these factors might not reflect some prodromal process but rather be risk factors or markers for risk factors. In the Swedish study, only personality disorders had an independent association with schizophrenia at 5 years. It will be important to establish whether non-psychotic disorders are anything more than reflections of the prodromal changes before the onset of schizophrenia.

Glyn Lewis, PhD, MRCPsych University of Bristol Bristol, UK

1 Lewis G, David AS, Malmberg A, et al. Non-psychotic psychiatric disorder and subsequent risk of schizophrenia. Cohort study. BrJ Psychiatry 2000;177:416-20. 